\title{
PARTICLE TRAPS
}

There was a time when a paper reporting a computer simulation might, in some fields (notably, molecular biology), be rejected purely on the grounds that it contained 'no experimental results' - simulation was seen as building castles in the air, disconnected from objective reality. Some now argue that, on the contrary, computer simulation is approaching the status of experiment, providing glimpses of what the real atoms and molecules in the corresponding material system are up to.

For non-practitioners, that latter view seems increasingly plausible. Surely the enormous increase in computing power must be enhancing the capabilities of simulation, allowing it to encompass bigger systems and to incorporate more of the nuances of molecular behaviour? It is sometimes whispered that we'll soon simulate a complete living cell or follow a crack all the way from atomic inception to the collapse of a bridge.

It's undoubtedly true that computer simulation can now handle systems of a complexity unimaginable when, for example, Nicholas Metropolis and his collaborators proposed a method in 1953 for sampling probability distributions in what became the Monte Carlo algorithm. But the allure of computational muscle for understanding molecules and materials has sometimes fooled researchers into imagining that sheer number-crunching offers a transparent and reliable predictive tool, a kind of virtual microscope for the molecular world.

Being misled by computer simulation is not necessarily a mark of naivety. Even experienced practitioners can be derailed by bugs, spotted only via an obstinate irreproducibility. But aside from coding glitches, simulations present a host of subtleties that may trip the unwary, as Daan Frenkel of Cambridge University explains in a recent preprint stemming from a summer school at Varenna in Italy (D. Frenkel, http://www.arxiv.org/ abs/1211.4440).

Frenkel's examination of the pitfalls - as he puts it, the "dark side" - of simulation offers many warnings that fellow experts know well. But in aggregate, they create a timely reminder, as the possibilities of computer simulation seem poised on the threshold of almost limitless potential, of what this sort of modelling truly entails: to put it bluntly, a cartoon version of molecular interactions that are themselves often imperfectly understood, hedged around with compromises yet offered in a proliferation of varieties whose relative merits may be poorly known.

Even well-explored issues such as boundary conditions may remain slippery. For example, structured

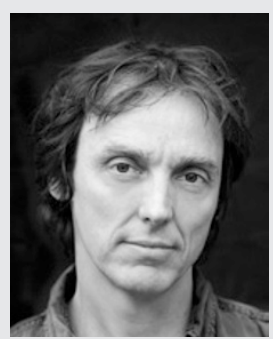

PHILIP BALL

materials such as smectic liquid crystals might find themselves confined to an inadequate configuration space unless the boundary conditions enable lateral interlayer exchange of particles. And a classic algorithm such as the 'particle insertion' method for determining chemical potentials is complicated for crystalline systems in which vacancies play a central role. It's all too tempting to assume, meanwhile, that molecular dynamics provides actual rather than merely possible time trajectories - a distinction particularly important for, say, investigating protein-folding mechanisms.

Beyond technical details, Frenkel supplies a selection of the myths that any field is liable to accumulate: what boundaries and free-energy landscapes do and do not mean, for instance.

Some of these myths are still matters of opinion, and Frenkel freely admits that a paper like this will contribute to them as well as explode them.

\section{Imaging cracks in hostile regimes}

The evolution of microcrack damage in materials under hostile thermal and mechanical conditions has now been imaged in three dimensions by real-time insitu X-ray microtomography.

\section{Philip J. Withers}

ur understanding of the physical world has progressed hand in hand with our ability to observe it. For instance, computer tomography (CT) which was originally developed for medical imaging - has been instrumental in the non-destructive testing of materials. In CT, a digital three-dimensional replica of the object under observation is reconstructed from hundreds or thousands of twodimensional projections (radiographs) collected at different illumination angles using X-rays, neutrons or other penetrating wave beams. Because the images are collected non-destructively, CT can be applied repeatedly to follow how the structure of a material changes over time. In fact, it is now possible to 